\title{
2.7 Machine Learning on Central Hemodynamic Quantities Using Noninvasive Measurements: How Far Can We Go?
}

\author{
Vasiliki Bikia*, Stamatia Pagoulatou, Nikolaos Stergiopulos
}

Swiss Federal Institute of Technology (EPFL), Lausanne, Switzerland

\section{ABSTRACT}

Background: Transforming peripheral noninvasive measurements to obtain central hemodynamic quantities, such as cardiac output $(\mathrm{CO})$ and central systolic blood pressure (cSBP), is a highly emerging field [1,2]. However, no holistic investigation has been performed to assess the amount of information contained in each peripheral measurement for the prediction of central values. This can be attributed to the inherent difficulty of creating a complete and accurate database; mainly due to the invasive nature of the gold standard techniques $[3,4]$.

Methods: To meet this need, we exploit synthetic data from a previously validated cardiovascular model (CVm) [5]. Our study relies on peripheral quantities including brachial pressure, heart rate (HR), and pulse wave velocity (PWV) simulated by the CVm. A Random Forest model was trained using 2744 synthetic instances and, subsequently, was tested against a subset of 800 . Correlations and feature importances of the input parameters were reported (Figure 1).

Results: Our results demonstrated that precise estimates of CO and CSBP were yielded with an RMSE of $0.39 \mathrm{~L} / \mathrm{min}$ and $1.39 \mathrm{mmHg}$, respectively (Figures 2 and 3). Low biases were observed, namely $0.03 \pm 0.39 \mathrm{~L} / \mathrm{min}$ for CO and $-0.08 \pm 1.39 \mathrm{mmHg}$ for cSBP. PWV, HR, and brachial pulse pressure were found to be the most correlated features with CO, whereas brachial SBP was plausibly shown to be the significant determinant of cSBP for our model (Figures 4 and 5).

Conclusion: These findings pave the way for better devising central hemodynamics' predictions. In the future, our ultimate goal is to examine the sensitivity of cardiac parameters estimation (i.e., elastance) to noninvasive peripheral measurements.

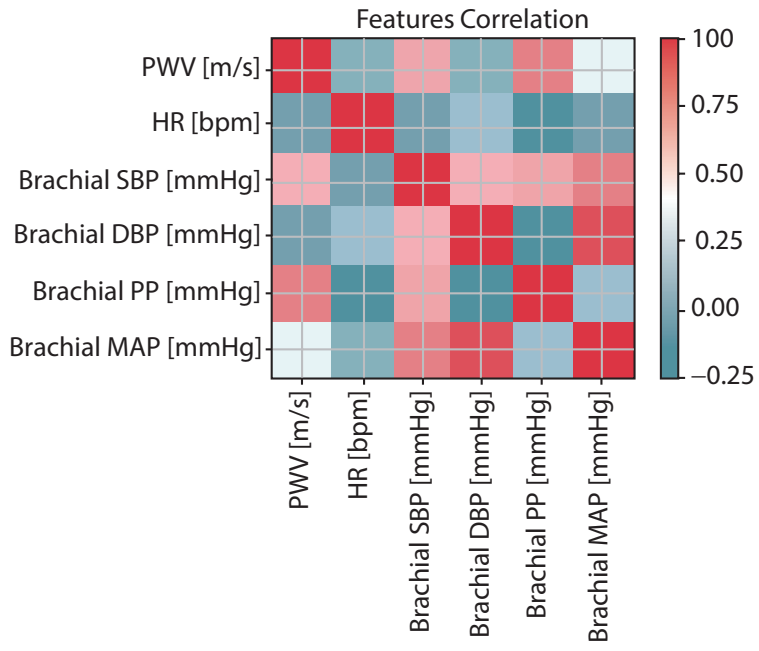

Figure 1 Correlation matrix. 
A
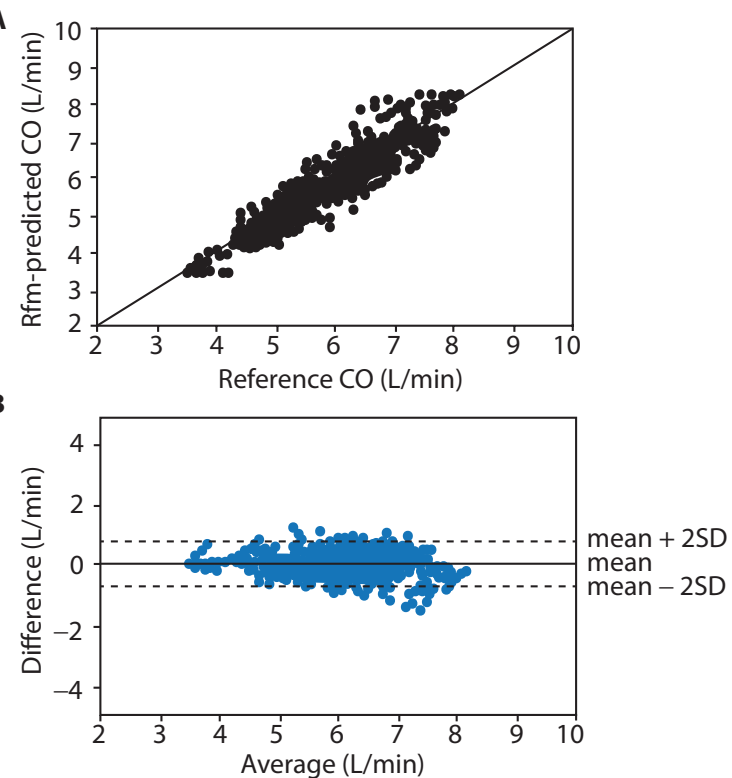

Figure 2 Scatterplot (A) and Bland-Altman plot (B) between predicted and reference CO values.

A
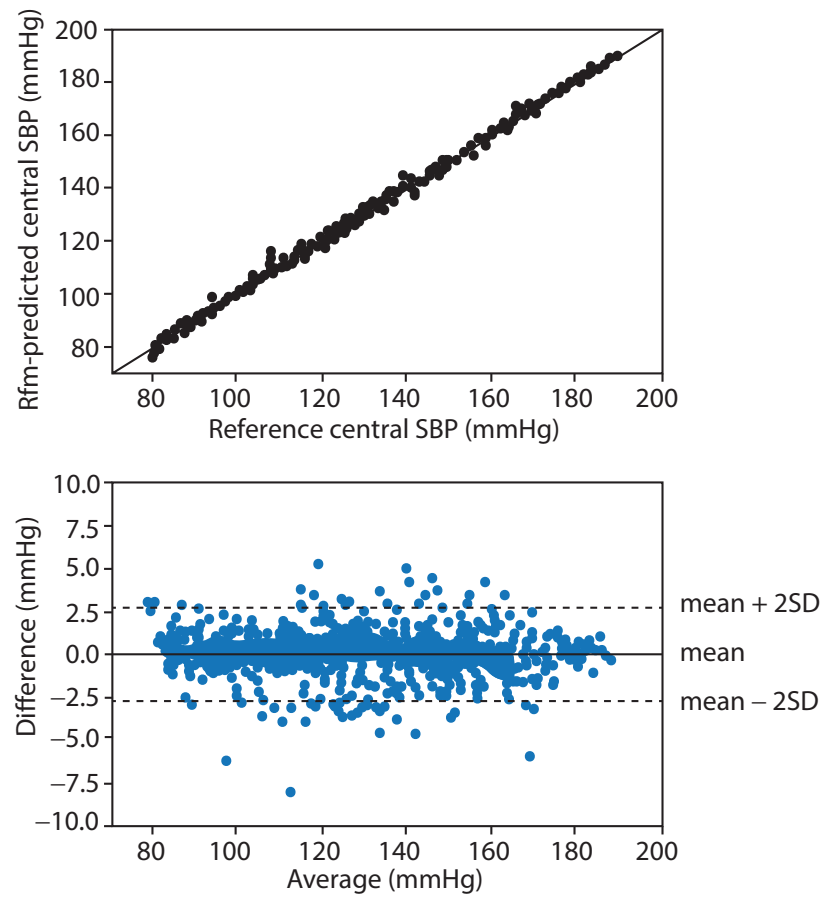

Figure 3 Scatterplot (A) and Bland-Altman plot (B) between predicted and reference CO values. 


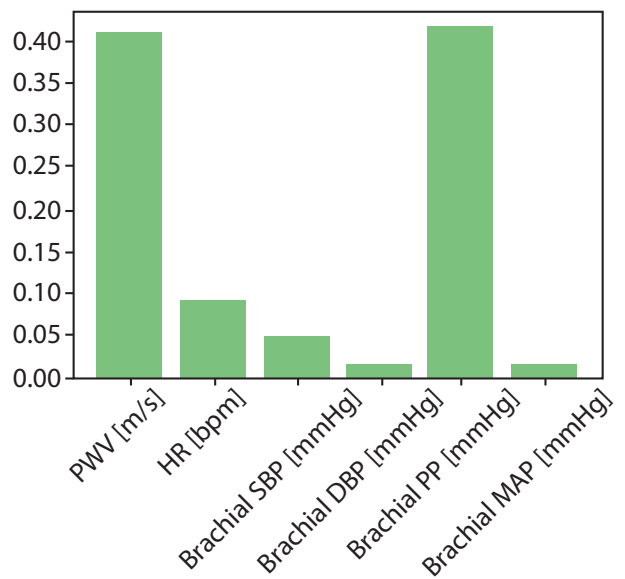

Figure 4 Feature importances for CO prediction.

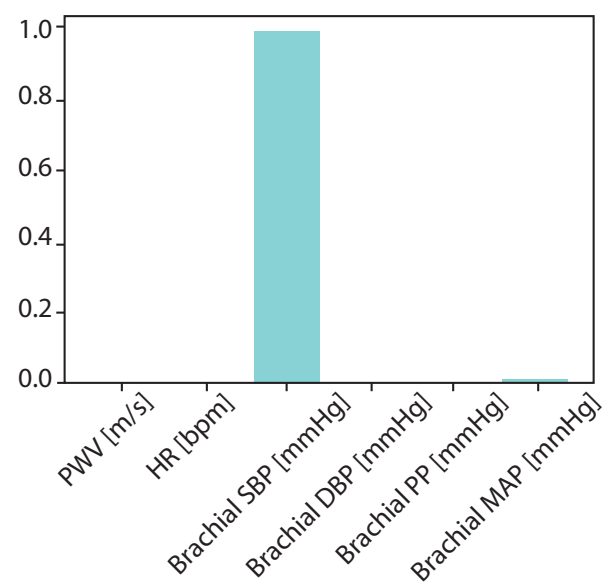

Figure 5 Feature importances for cSBP.

\section{REFERENCES}

[1] Hope SA, Tay DB, Meredith IT, Cameron JD. Use of arterial transfer functions for the derivation of aortic waveform characteristics. J Hypertens 2003;21:1299-305.

[2] Swamy G, Mukkamala R. Estimation of the aortic pressure waveform and beat-to-beat relative cardiac output changes from multiple peripheral artery pressure waveforms. IEEE Trans Biomed Eng 2008;55:1521-9.

[3] Avolio A. Central aortic blood pressure and cardiovascular risk: a paradigm shift? Hypertension 2008;51:1470-1.

[4] Jansen JRC, Schreuder JJ, Mulier JP, Smith NT, Settels JJ, Wesseling KH. A comparison of cardiac output derived from the arterial pressure wave against thermodilution in cardiac surgery patients. Br J Anaesth 2001;87:212-22.

[5] Reymond P, Merenda F, Perren F, Rüfenacht D, Stergiopulos N. Validation of a one-dimensional model of the systemic arterial tree. Am J Physiol Heart Circ Physiol 2009;297:H208-H22.

(c) 2019 Association for Research into Arterial Structure and Physiology. Publishing services by Atlantis Press International B.V. This is an open access article distributed under the CC BY-NC 4.0 license (http://creativecommons.org/licenses/by-nc/4.0/). 